\title{
Intermittent random walks for an optimal search strategy: One-dimensional case
}

\author{
G.Oshanin ${ }^{1}$, H.S.Wio ${ }^{2}$, K.Lindenberg ${ }^{3}$, and S.F.Burlatsky ${ }^{4}$ \\ ${ }^{1}$ Physique Théorique de la Matière Condensée (UMR 7600), Université Pierre et \\ Marie Curie - Paris 6, 4 place Jussieu, 75252 Paris France and Department of \\ Inhomogeneous Condensed Matter Theory, Max-Planck-Institute für \\ Metallforschung, Heisenbergstrasse 3, D-70569 Stuttgart, Germany \\ ${ }^{2}$ Instituto de Fisica de Cantabria, Avda. Los Castros s/n, E-39005 Santander, Spain \\ ${ }^{3}$ Department of Chemistry and Biochemistry 0340 and Institute for Nonlinear \\ Science, University of California, San Diego, La Jolla, CA 92093-0340, USA \\ ${ }^{4}$ United Technologies Research Center, United Technologies Corp., 411 Silver Lane, \\ 129-21 East Hartford, CT 06108, USA \\ E-mail: oshanin@lptl.jussieu.fr, wio@ifca.unican.es, \\ kl@hypatia.ucsd.edu, burlatsf@utrc.utc.com
}

\begin{abstract}
We study the search kinetics of an immobile target by a concentration of randomly moving searchers. The object of the study is to optimize the probability of detection within the constraints of our model. The target is hidden on a onedimensional lattice in the sense that searchers have no a priori information about where it is, and may detect it only upon encounter. The searchers perform random walks in discrete time $n=0,1,2, \ldots, N$, where $N$ is the maximal time the search process is allowed to run. With probability $\alpha$ the searchers step on a nearest-neighbour, and with probability $(1-\alpha)$ they leave the lattice and stay off until they land back on the lattice at a fixed distance $L$ away from the departure point. The random walk is thus intermittent. We calculate the probability $P_{N}$ that the target remains undetected up to the maximal search time $N$, and seek to minimize this probability. We find that $P_{N}$ is a non-monotonic function of $\alpha$, and show that there is an optimal choice $\alpha_{\text {opt }}(N)$ of $\alpha$ well within the intermittent regime, $0<\alpha_{\text {opt }}(N)<1$, whereby $P_{N}$ can be orders of magnitude smaller compared to the "pure" random walk cases $\alpha=0$ and $\alpha=1$.

PACS numbers: 87.23.-n, 05.40.-a
\end{abstract}

Submitted to: J. Phys. C: Solid State Phys. 


\section{Introduction}

Everybody searches for something: predators look for prey, prey forage, human beings look for better places to work or for lost keys or for partners, spies search for hidden secrets, and knights desperately seek the Holy Grail, whatever it may be.

The search for a desired target may be long and uncertain; targets may be sparse, hidden or difficult to detect even when found, or they may have their own life-time and vanish before they are detected. That is why efficient search strategies appropriate to specific types of situations are highly desirable. The question of optimal search strategies has motivated a great deal of work within the last years [1-12].

While earlier work has focused on systematic searches in organized human activities such as rescue operations [1-4], more recent analyses have been devoted to random strategies [5-11]. In particular, it has been realized that in the case of the so-called non-destructive search, when the target reappears after some time at the same location, and when the searcher always remains within the system, Lévy flights with randomly reoriented ballistic trajectories provide an optimal search efficiency [5-9]. On the other hand, following the observation of trajectories of foraging animals such as lizards or fish or birds characterized by two distinct types of motion - fast relocation stages non receptive to the target and relatively slow reactive phases when the target may be detected $[13,14]$ - another type of random search, an intermittent search, has been proposed. Analytical modeling of such random intermittent search strategies has been put forth in [11] (see also [12]).

In this paper we discuss the search of a single target hidden on a one-dimensional regular lattice by a concentration of searchers performing intermittent random walks. In particular, at each tick of the clock each of the searchers chooses between two possibilities. One is to jump to a nearest-neighbouring lattice site, and the other is to leave the lattice and fly with a given velocity until it lands back on the lattice at a fixed distance $L$ from the departure site. The term "hidden" means that the searchers can detect the target only upon landing directly on the target site at the end of a flight, or via a one-step (nearest-neighbour) walk onto that site. Intermittency has been invoked in other models (e.g., see the models proposed in [11,12]) and has been observed in a number of ecological and other contexts (see, e.g., [13,14]), but all the other features of our model are, to the best of our knowledge, new.

Contrary to previous work, which focused on the analysis and optimization of the first passage time to the target from a given location, or on the number of targets encountered [5-11], here we study the behaviour of a different property, namely, the

probability that a single target remains undetected up to the maximal search time $N$. We can not, of course, make this random search process certain; that is, we can not guarantee that the target is found (or not found) with unit probability in a finite time, but what we are able to show is that the non-detection probability is a non-monotonic function of the parameter $\alpha$ which determines whether a searcher takes a nearest-neighbour step (probability $\alpha$ ) or a long flight [probability $(1-\alpha)$ ], and that it has a sharp minimum at 
some value of $\alpha$. Consequently, we show that the search efficiency can be dramatically enhanced, by orders of magnitude, by choosing an appropriate value of $\alpha$.

The outline of this paper is as follows. In section 2 we describe our model of intermittent random walks, present definitions, and delineate our main results. In section 3 we derive a general formula for the non-detection probability. Section 4 is devoted to the analysis of different properties of the intermittent random walk. In section 5 we focus on a particular exactly solvable case, namely, a Lindenberg-Shuler intermittent walk with steps of unit length and flights of two lattice spacings. For this example we discuss the specific features of the optimization procedure. Next, in section 6 we consider the general case of intermittent random walks with steps of unit length and flights of fixed length $L$, and define an optimal search strategy. Finally, in section 7 we conclude with a brief summary of our results and an outlook of future work.

\section{The model, basic notation and results}

Consider a one-dimensional regular lattice of unit spacing containing $M+1$ sites $s$. This is the substrate. At one of the lattice sites, say at the origin $s=0$, we hide an immobile target, and only we know that it is there. Then, we randomly place $K$ searchers in the lattice under the constraint that none of them is placed at the site with the hidden target, that is, we distribute them randomly over the remaining $M$ sites. The searchers have no knowledge of the location of the target.

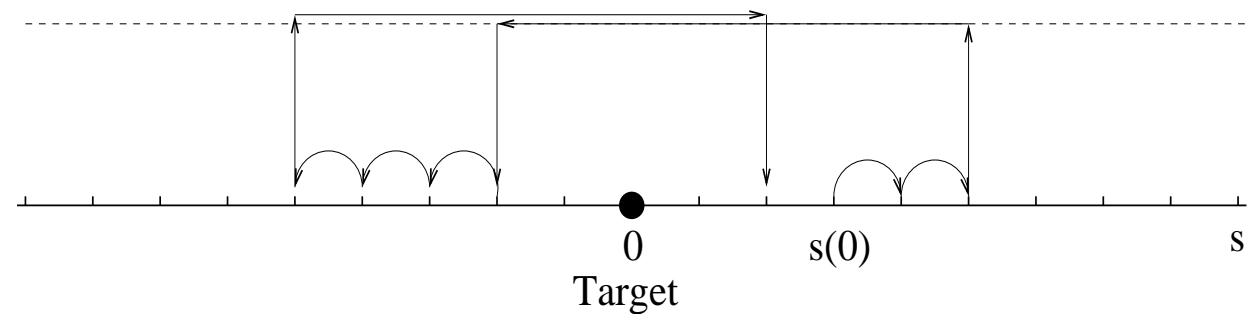

Figure 1. A sketch of a 7-step trajectory of an intermittent random walk, starting at $s(0)$, with nearest-neighbor steps (probability $\alpha$ ) and off-lattice flights [probability $(1-\alpha)$ ] over a distance $L$ (here, $L=7)$. The arrows indicate the step direction. Note that this trajectory does not find the target.

Next, we let the searchers move according to the following rule (see figure 11): At each tick of the clock, $n=1,2,3, \ldots, N$ ( $N$ is the maximal time the search process may run), each searcher selects randomly between two possibilities. With probability $\alpha$, the searcher jumps to one of its nearest neighbouring sites, with equal probabilities to the right or to the left. With probability $(1-\alpha)$, the searcher leaves the lattice and flies off-lattice with a given velocity $V$ until it lands at a site an fixed integer distance $L$ away from the departure site. The direction of the flight is chosen at random, with equal probabilities to right or left. The time the searcher spends off-lattice during a flight is $T=L / V$, a model parameter that can have integer values $T=1,2, \ldots, L$. Note that 
this value defines the velocity $V$.

As stated earlier, the term "hidden" means that a searcher can not perceive the target when it is off-lattice and may detect it only when it lands on the target site. The searcher may land there at the end of a flight or by a one-step jump. Once the searcher lands on the target site, one can define an elementary detection probability $q$. The target may immediately be recognized when first encountered by a searcher $(q \equiv 1$, perfect detection), or detection may take place with a given probability $0<q<1$. In the latter case, when any of the searchers arrives at the site occupied by the target, there is always a possibility that the target remains undetected (imperfect detection). Here we focus on the perfect detection case. Some aspects of the search kinetics in the imperfect detection case for the one-dimensional system will be discussed briefly in section 7 . An extension of the model to $d$-dimensional spaces and some other subtle questions not pursued here will be discussed elsewhere [15].

Our first goal is to determine the probability $P_{N}$ that the target remains undetected up to time $N$, the maximal time the search process is allowed to run, which merely depends on our patience or on experimental constraints. We show in the next section that in the perfect detection limit the probability $P_{N}$ that no searcher has reached the target up to time $N$ obeys the standard formula (see, e.g. $[16,17]$ for more details)

$$
P_{N}=\exp \left(-\rho S_{N}\right),
$$

where $S_{N}$ is the expected value of the number of distinct sites visited on the substrate by any searcher in an $N$-step walk (see, e.g., [18]). This is a crucial equation, since we will arrive at results for $P_{N}$ via calculations of $S_{N}$.

We will calculate $S_{N}$ explicitly for the intermittent random walk and determine its asymptotic behaviour analytically in the large- $N$ limit. Using these results, we set out to show that there always exists a value $\alpha=\alpha_{\text {opt }}(N), 0<\alpha_{\text {opt }}(N)<1$, which maximizes the expected number of distinct substrate sites visited by an intermittent random walk up to time $N$. Consequently, it minimizes the probability $P_{N}$ in equation (2.1). We demonstrate that by choosing this optimal value of $\alpha$ the non-detection probability $P_{N}$ in equation (2.1) can be made orders of magnitude smaller than those of the "pure" cases $\alpha=1$ (nearest-neighbor steps only) or $\alpha=0$ (flights only), for which asymptotically

$$
P_{N}(\alpha=1)=\exp \left[-\rho\left(\frac{8 N}{\pi}\right)^{1 / 2}+\mathcal{O}\left(\frac{1}{\sqrt{N}}\right)\right] .
$$

and

$$
P_{N}(\alpha=0)=\exp \left[-\rho\left(\frac{8 N}{\pi T}\right)^{1 / 2}+\mathcal{O}\left(\frac{1}{\sqrt{N}}\right)\right],
$$

The result (2.2) is well-known and reflects the fact that a random walk in low dimensional systems $(d=1$ and $d=2)$ compactly explores the space; that is, $S_{N}$ grows sublinearly with time $N$, and each site ever visited by such a walk is most probably visited many times. Hence, searching a target in a low dimensional system with the help of a standard random walk is not very efficient, since the walker wastes a lot of time revisiting sites 
that do not contain the target. The result (2.2) is the same with the replacement $N \rightarrow N / T$ (i.e., it is the same as that of a nearest neighbor random walk for a shorter time). This further decreases the probability of detection because it does not explore the local regions at all before making a jump to a site that has probably been visited many times before. It is thus not an efficient search strategy either. We find that although the sublinear growth of $S_{N}$ is unavoidable in any one dimensional random walk, and, even more specifically, the asymptotic dependence $S_{N} \propto N^{1 / 2}$, for fixed $L$ it is possible to optimize the search strategy via an intermittent walk with a particular choice of $\alpha$ that depends on the length of the walk (calculated explicitly later). We obtain the asymptotic result

$$
P_{N}\left(\alpha=\alpha_{\text {opt }}(N)\right) \sim \exp \left[-\rho(L V)^{1 / 2}\left(\frac{8 N}{\pi}\right)^{1 / 2}+\rho \gamma(L)\left(\frac{8 N}{\pi}\right)^{1 / 6}+o\left(N^{1 / 6}\right)\right]
$$

where $\gamma(L)$ is an increasing function of the flight distance $L$. Note that the leading term in the exponent contains a pre-factor $(L V)^{1 / 2}, V=1,2,3, \ldots, L$, which can make it considerably larger than the corresponding terms in equations (2.3) and (2.2) and thus may result in a substantial increase of the detection probability.

\section{Non-detection probability}

In this section we define the probability $P_{N}$ that the target is not detected up to time $N$, and express it using well-known properties of random motion that have been extensively discussed in the literature (see, e.g., $[17,18]$ and references therein). In particular, we begin by introducing the notation $s(n)$ to denote the random process that describes a searcher's trajectory, and $P(s \mid s(0) ; n)$ to denote the probability that $s(n)$, which is initially at site $s(0)$, is at site $s$ at time $n$. We find it useful to present the derivation because, contrary to most studies, we are interested in defining the number of distinct sites visited by $s(n)$ only on the substrate, which is a subset of all available space. Indeed, most existing work concerns random walks that never leave the lattice so that the equality $\sum_{s} P(s \mid s(0) ; n) \equiv 1$ holds at all times. In our case this probability is not conserved, $\sum_{s} P(s \mid s(0) ; n)<1$, since the searchers spend some portion of their time off-lattice. We present this analysis in the most general case, assuming only that $s(n)$ is a homogeneous process.

Suppose there are altogether $K$ searchers labeled $k=1,2, \ldots, K$, and let $s_{k}(n)$ denote a given $n$-step trajectory of the $k$-th searcher. Then, in the perfect detection case the indicator function $\Psi\left(\left\{s_{k}(N)\right\}\right)$ of the event that the target has not been detected up to time $N$ for (arbitrary) given trajectories of all $K$ searchers can be written as

$$
\Psi\left(\left\{s_{k}(N)\right\}\right)=\prod_{k=1}^{K} \prod_{n=0}^{N}\left[1-I\left(s_{k}(n)\right)\right], \quad I(s)= \begin{cases}1, & s=0 \\ 0, & s \neq 0\end{cases}
$$

This indicator function has the correct behavior, namely, for this particular set of trajectories $\Psi=0$ if any searcher hits the target and $\Psi=1$ if none do. All searchers are 
assumed to move independently. Averaging $\Psi\left(\left\{s_{k}(N)\right\}\right)$ over all trajectories and initial positions thus yields the probability $P_{N}$ that none of the searchers has ever reached the site $s=0$ up to time $N$,

$$
\begin{aligned}
P_{N} & =\left[\frac{1}{M} \sum_{s(0)}^{\prime} E_{s(0)}\left\{\prod_{n=0}^{N}[1-I(s(n))]\right\}\right]^{K} \\
& =\left[1-\frac{1}{M} \sum_{s(0)}^{\prime} E_{s(0)}\left\{1-\prod_{n=0}^{N}(1-I(s(n)))\right\}\right]^{K},
\end{aligned}
$$

where $E_{s(0)}\{\ldots\}$ stands for the average over all trajectories starting at site $s(0)$, and the prime on the sum indicates the exclusion of the target site $s(0)=0$. Note that the summation extends over all non-target sites of the substrate, a one-dimensional lattice, and does not include off-lattice contributions. In the thermodynamic limit we set $K, M \rightarrow \infty$ keeping their ratio fixed, $K / M=\rho=$ density of searchers, to obtain

$$
P_{N}=\exp \left[-\rho \sum_{s(0)}^{\prime} E_{s(0)}\left\{1-\prod_{n=0}^{N}[1-I(s(n))]\right\}\right] .
$$

Since $s(n)$ is a homogenous process, equation (3.3) can be rewritten as

$$
P_{N}=\exp \left[-\rho E_{0}\left\{\sum_{s=1}^{\infty}\left(1-\prod_{n=0}^{N}\left[1-I\left(s^{\prime}(n)-s\right)\right)\right]\right\}\right],
$$

where now $E_{0}\{\ldots\}$ denotes an average over trajectories of an auxiliary process $s^{\prime}(n)=$ $s(n)-s(0)$ which starts at the origin at $n=0$. Note that the indicator function $\left(1-\prod_{n=0}^{N}\left[1-I\left(s^{\prime}(n)-s\right)\right]\right)$ shows whether the site $s$ on the substrate has ever been visited by the process $s^{\prime}(n), n=0,1,2, \ldots, N$. Consequently, the sum over all substrate sites is just the realization-dependent number of distinct sites visited by an individual searcher, and its average leads directly to the crucial equation (2.1).

For this proof to be useful in calculating $P_{N}$ we next need to calculate $S_{N}$. For this purpose, note that the function in the exponent of (3.3) $),\left\{1-\prod_{n=0}^{N}[1-I(s(n))]\right\}$, is the indicator function of the event that $s(N)$ has at least once visited the origin, so that

$$
\sum_{s(0)}^{\prime} E_{s(0)}\left\{1-\prod_{n=0}^{N}[1-I(s(n))]\right\}=\sum_{s(0)}^{\prime} R_{N}(0 \mid s(0)),
$$

where $R_{N}(0 \mid s(0))$ is the probability that an arbitrary $N$-step random process $s(N)$ has visited the origin at least once. We can therefore write

$$
P_{N}=\exp \left(-\rho \sum_{s(0)}^{\prime} R_{N}(0 \mid s(0))\right) \text {. }
$$

Next we note that, by definition, $R_{N}(0 \mid s(0))$ can be formally represented as

$$
R_{N}(0 \mid s(0))=\sum_{n=0}^{N} F_{n}(0 \mid s(0))
$$


where $F_{n}(0 \mid s(0))$ is the probability that $s(n)$ arrived at the origin for the first time on the $n$-th step, given that it started at site $s(0)$. Applying the theory of recurrent events (see, e.g., [18]), the event that $s(n)$ is at site 0 after $n$ steps can be decomposed into the $n$ mutually exclusive events " $s(n)$ first arrived at site 0 after $j$ steps, and subsequently returned to site 0 in $(n-j)$ steps." This allows us to write for the distribution function $P(0 \mid s(0) ; n)$ the discrete "integral equation"

$$
P(0 \mid s(0) ; n)=\delta_{0, s(0)} \delta_{n, 0}+\sum_{j=1}^{n} F_{j}(0 \mid s(0)) P(0 \mid 0 ; n-j),
$$

which implies that the generating functions

$$
\begin{aligned}
& P(s \mid s(0) ; z)=\sum_{n=0}^{\infty} P(s \mid s(0) ; n) z^{n}, \\
& F(s \mid s(0) ; z)=\sum_{n=0}^{\infty} F_{n}(s \mid s(0)) z^{n}
\end{aligned}
$$

at $s=0$ are simply related to each other,

$$
F(0 \mid s(0) ; z)=P(0 \mid s(0) ; z)-\frac{\delta_{0, s(0)}}{P(0 \mid 0 ; z)} \text {. }
$$

It then follows that

$$
R(0 \mid s(0) ; z)=\sum_{n=0}^{\infty} R_{N}(0 \mid s(0)) z^{N}=\frac{1}{1-z} F(0 \mid s(0) ; z),
$$

and

$$
S(z)=\sum_{n=0}^{\infty} S_{N} z^{N}=\sum_{s(0)}^{\prime} R(0 \mid s(0) ; z)=\frac{1}{1-z} \frac{\sum_{s} P(s \mid 0 ; z)}{P(0 \mid 0 ; z)} .
$$

Hence, in order to determine $P_{N}$ in equation (2.1) via $S(z)$ we only need to determine $P(0 \mid 0 ; z)$ and the normalization $\sum_{s} P(s \mid 0 ; z)$. We proceed with this determination in the next section.

\section{Properties of the intermittent random walk}

Since the searchers in our model move independently, it suffices to focus on the properties of the walk of an individual searcher. The probability $P(s \mid s(0) ; n)$ of being at site $s$ after $n$ steps obeys the recurrence relation

$$
\begin{aligned}
P(s \mid s(0) ; n)= & \frac{\alpha}{2}[P(s-1 \mid s(0) ; n-1)+P(s+1 \mid s(0) ; n-1)] \\
& +\frac{(1-\alpha)}{2}[P(s-L \mid s(0) ; n-T)+P(s+L \mid s(0) ; n-T)]
\end{aligned}
$$

for $n=0,1,2, \ldots, N$. Jumps between nearest-neighbouring sites occur in one unit of time, while long-range jumps over distance $L$ require an integer time $T$. Equation (4.1) therefore defines a non-Markovian process with a memory. Since the intermittent 
random walks are homogeneous so that $P(s \mid s(0) ; n)=P(s-s(0) \mid 0 ; n)$, without loss of generality we henceforth set $s(0)=0$.

The Fourier-transformed generating function

$$
\Phi(k, z)=\sum_{s} \exp (i k s) P(s \mid 0 ; z)
$$

can easily be calculated by multiplying both sides of equation (4.1) by $\exp (i k s)$ and $z^{n}$ and summing over all substrate sites $s$ and time $n$. We readily find that

$$
\Phi(k, z)=\left[1-\alpha z \cos (k)-(1-\alpha) z^{T} \cos (k L)\right]^{-1} .
$$

Consequently, the lattice Green function (or site occupation generating function) of the intermittent walk is given by

$$
P(s \mid 0 ; z)=\frac{1}{\pi} \int_{0}^{\pi} \frac{\cos (k s) d k}{1-\alpha z \cos (k)-(1-\alpha) z^{T} \cos (k L)} .
$$

One of the two quantities required for $S(z)$ in equation (3.12) follows immediately from equations (4.2) and (4.3) by setting $k=0$,

$$
\sum_{s} P(0 \mid s ; z)=\Phi(k=0, z)=\left(1-\alpha z-(1-\alpha) z^{T}\right)^{-1} .
$$

This leaves only the evaluation of $P(0 \mid 0 ; z)$ for the calculation of $S(z)$ and consequently of $S_{N}$ and $P_{N}$ [cf. equation (2.1) ]. We shall return to this calculation and to our goal of maximizing the detection probability of the target presently, but first we briefly digress to obtain some associated interesting properties of the underlying distribution $P(s \mid 0 ; n)$.

\subsection{Second and fourth moments of the distribution $P(s \mid 0 ; n)$}

Although we do not use them directly in the evaluation of $P_{N}$, it is instructive to explicitly consider second and the fourth moments of the distribution function $P(s \mid 0 ; n)$ because they provide some insight into some aspects of optimal behavior.

Consider the second moment of the distribution. One expects that at long times the mean square displacement of a particle will be diffusive, that is, that the second moment $\overline{s^{2}(n)}$ for a particle starting from the origin should grow proportionally with time $n$. The overline denotes an average over many trajectories. We differentiate $\Phi(k, z)$ of Eq.(4.3) twice with respect to $k$ and set $k=0$ to find that the generating function of the second moment obeys

$$
\overline{s^{2}(z)}=\sum_{n=0}^{\infty} z^{n} \overline{s^{2}(n)}=\frac{\alpha z+(1-\alpha) z^{T} L^{2}}{\left(1-\alpha z-(1-\alpha) z^{T}\right)^{2}} .
$$

As $z \rightarrow 1^{-}$the leading contribution to the generating function is of order $\mathcal{O}\left[(1-z)^{-2}\right]$, which leads to linear growth in time, $\overline{s^{2}(n)}=2 D n$, with diffusion coefficient

$$
D=\frac{1}{2} \frac{\alpha+(1-\alpha) L^{2}}{(\alpha+(1-\alpha) T)^{2}} .
$$

Note that for $\alpha=1$ and $\alpha=0$ this reduces respectively to the standard results $D=1 / 2$ and $D=V^{2} / 2$. 
One must now distinguish between two situations, the cases $T=1$ and $T>1$. In the first case, when the flight over distance $L$ requires one unit of time precisely as does a nearest-neighbour step, $D$ is a monotonically decreasing function of $\alpha$. It is largest when $\alpha=0$, when all jumps are flights over a distance $L$ requiring a unit of time, and achieves its minimal value $1 / 2$ when $\alpha=1$, when there are no long-range flights. This is, of course, the behaviour one would expect since in the case $T=1$ the particle is always on the lattice. On the other hand, when $T>1$ and the particle spends some portion of its time off-lattice, the situation is completely different. Here, D in equation (4.7) shows a non-monotonic behaviour as a function of $\alpha$. Differentiating Eq.(4.7) with respect to $\alpha$, we find that $D$ has a maximum when

$$
\alpha=\alpha^{(2)}=\frac{T+L^{2}(T-2)}{(T-1)\left(L^{2}-1\right)},
$$

where the superscript " 2 " stresses that this value of $\alpha$ only corresponds to the maximum of the second moment and not necessarily of other moments. Note that $0<\alpha^{(2)}<1$, which implies that here, contrary to the case $T=1$, the greatest mean square displacement is achieved by an intermittent random walk, that is, one that includes nearest-neighbour steps as well as off-lattice flights over a distance $L$. The value of $D$ corresponding to $\alpha=\alpha^{(2)}$ is given by

$$
D_{\max }=\frac{1}{8} \frac{\left(L^{2}-1\right)^{2}}{(T-1)\left(L^{2}-T\right)}
$$

For large $L, D_{\max }$ may be made very large since $D_{\max } \sim L^{2} / T=L V$. On the other hand, there is a penalty to pay, since $\overline{s^{2}(n)}=2 D n$ is an asymptotic result which holds only for sufficiently long times that increases with increasing $L$. By increasing $L$ and optimizing $\alpha$ we can thus make $D$ arbitrarily large, but to observe this regime we will have to wait a progressively longer time.

Consider next the fourth moment $\overline{s^{4}(n)}$ of the distribution function. Its generating function obeys

$\overline{s^{4}(z)}=\sum_{n=0}^{\infty} z^{n} \overline{s^{4}(n)}=\frac{6\left(\alpha z+(1-\alpha) L^{2} z^{T}\right)^{2}}{\left(1-\alpha z-(1-\alpha) z^{T}\right)^{3}}+\frac{\alpha z+(1-\alpha) L^{4} z^{T}}{\left(1-\alpha z-(1-\alpha) z^{T}\right)^{2}}$.

The leading behavior of the generating function near $z \rightarrow 1^{-}$is of order $\mathcal{O}\left[(1-z)^{-3}\right]$, so that at sufficiently long times $\overline{s^{4}(n)}$ is quadratic in $n$,

$$
\overline{s^{4}(n)} \sim \frac{3\left(\alpha+(1-\alpha) L^{2}\right)^{2}}{(\alpha+(1-\alpha) T)^{3}} n^{2} .
$$

We point out again that the coefficient of $n^{2}$ shows a completely different behavior depending on whether $T=1$ or $T>1$. In the former case, when particles spend all of their time on the lattice, it is a monotonic function of $\alpha$ with a maximum at $\alpha=0$. On the other hand, for $T>1$ the coefficient is again a non-monotonic function of $\alpha$. The maximum is achieved at

$$
\alpha^{(4)}=\frac{2 T+L^{2}(T-3)}{(T-1)\left(L^{2}-1\right)} .
$$


For $T>2$, the extremum $\alpha^{(4)}$ is also well within the intermittent regime.

Note that $\alpha^{(4)} \neq \alpha^{(2)}$, (or more precisely, $\alpha^{(4)}<\alpha^{(2)}$ ). This alerts us to the fact that there is not a single "optimal choice" of $\alpha$. The choice clearly depends on the property or strategy that one wishes to optimize, and for more complex properties it is expected to depend on the maximal search time $N$. We are clear on the particular strategy that we wish to optimize, namely, the probability that the target will be detected by at least one of the searchers by time $N$. It is unlikely that either $\alpha^{(2)}$ or $\alpha^{(4)}$ provide this optimization. Indeed, we find below that the optimal detection strategy is achieved with an $N$-dependent value of $\alpha$ that also depends on the jump velocity.

\subsection{Probability of return to the origin and expected number of distinct sites visited}

We now return to the calculation at the end of section 4 to find the generating function of the expected value of the number of distinct sites visited by an individual searcher. With the substitution of equation (4.5) into equation (3.12), this generating function is given by

$$
S(z)=\frac{1}{(1-z)\left(1-\alpha z-(1-\alpha) z^{T}\right)} \frac{1}{P(0 \mid 0 ; z)} .
$$

We thus turn to the analysis of $P(0 \mid 0 ; z)$.

We start by noting that equation (4.4) can be written as

$$
P(s \mid 0 ; z)=\int_{0}^{\infty} d t e^{-t} I_{s}\left(\zeta_{1}, \zeta_{L}\right),
$$

where $I_{s}\left(\zeta_{1}, \zeta_{L}\right)$ is a particular two-variable case of a multi-variable generalized modified Bessel function [19],

$$
I_{s}\left(\zeta_{1}, \zeta_{2}, \ldots\right)=\frac{1}{\pi} \int_{0}^{\pi} d k \cos (k s) \exp \left[\sum_{m=1}^{\infty} \zeta_{m} \cos (m k)\right],
$$

in which $\zeta_{1}=\alpha z t, \zeta_{L}=(1-\alpha) z^{T} t$, and all other $\zeta_{m}$ are zero. Furthermore, $I_{0}\left(\zeta_{1}, \zeta_{L}\right)$ obeys

$$
I_{0}\left(\zeta_{1}, \zeta_{L}\right)=I_{0}(\alpha z t) I_{0}\left((1-\alpha) z^{T} t\right)+2 \sum_{l=1}^{\infty} I_{l L}(\alpha z t) I_{l}\left((1-\alpha) z^{T} t\right) .
$$

Subsitituting this latter expansion into the integral on the right-hand-side of equation (4.14) with $s=0$, performing the integration over $t$, and using the integral representation

$$
I_{n}(p)=\frac{(-1)^{n}}{\pi} \int_{-1}^{1} \frac{d x}{\sqrt{1-x^{2}}} e^{-p x} T_{n}(x),
$$

where the $T_{n}(x)$ are Tchebychev polynomials of the first kind, we find that occupation generating function for the site $s=0$ reads

$P(0 \mid 0, z)=\frac{1}{\pi^{2}} \int_{-1}^{1} \frac{d x}{\sqrt{1-x^{2}}} \int_{-1}^{1} \frac{d y}{\sqrt{1-y^{2}}} \frac{\left[1+2 \sum_{l=1}^{\infty}(-1)^{L l+l} T_{L l}(x) T_{l}(y)\right]}{1+\alpha z x+(1-\alpha) z^{T} y}$. 
Next, expanding the kernel in a series of Tchebychev polynomials,

$$
\frac{1}{1+\alpha z x+(1-\alpha) z^{T} y}=\frac{1}{\alpha z \sqrt{\tau^{2}-1}}\left[1+2 \sum_{n=1}^{\infty}(-\xi)^{n} T_{n}(x)\right]
$$

where

$$
\tau=\tau(y)=\frac{1+(1-\alpha) z^{T} y}{\alpha z} \geq 1, \quad \xi=\tau-\sqrt{\tau^{2}-1}
$$

we obtain a closed-form expression for $P(0 \mid 0, z)$ convenient for further analysis,

$$
P(0 \mid 0 ; z)=\frac{1}{\pi \alpha z} \int_{-1}^{1} \frac{d y}{\sqrt{1-y^{2}} \sqrt{\tau^{2}-1}} \frac{1-\xi^{2 L}}{1+2 \xi^{L} y+\xi^{2 L}} .
$$

Note that it is straightforward to perform the integral in equation (4.21) in the two "pure" limits $\alpha=1$ and $\alpha=0$. In the first case we have $\tau=1 / z, \xi=\left(1-\sqrt{1-z^{2}}\right) / z$, and

$P_{\alpha=1}(0 \mid 0 ; z)=\frac{1}{\pi \sqrt{1-z^{2}}} \int_{-1}^{1} \frac{T_{0}(y) d y}{\sqrt{1-y^{2}}}\left[1+2 \sum_{l=1}^{\infty}\left(-\xi^{L}\right)^{l} T_{l}(y)\right]=\frac{1}{\sqrt{1-z^{2}}}$.

On the other hand, when $\alpha=0$, we have $\tau=\infty, \xi=0$, and

$$
P_{\alpha=0}(0 \mid 0 ; z)=\frac{1}{\pi} \int_{-1}^{1} \frac{d y}{\sqrt{1-y^{2}}} \frac{1}{1+z^{T} y}=\frac{1}{\sqrt{1-z^{2 T}}} .
$$

We notice that for $T=1$ the two latter expressions coincide. One might therefore expect that $P(0 \mid 0 ; z)$ and the expected number of distinct sites visited will be non-monotonic functions of $\alpha$.

In the next two sections we explicitly calculate $P(0 \mid 0 ; z)$ and ultimately the desired target detection probability for intermittent walks.

\section{Particular case: Lindenberg-Shuler intermittent random walks with $\mathrm{L}=\mathbf{2}$ and $\mathrm{T}=1,2$}

To highlight the optimization procedure, consider first the simple case of intermittent random walks with nearest-neighbour steps and jumps over a distance $L=2$, first studied by Lindenberg and Shuler in [20] and [21]. In this case the integrals in equations (4.4) and (4.21) can be performed explicitly. For $L=2$ (and $T=1$ or $T=2$ ), the generating function for the probability of being at the origin is given by

$P(0 \mid 0 ; z)=\frac{1}{\left[1+(1-\alpha) z^{T}\right]}\left(\frac{\lambda_{2}}{\lambda_{2}-\lambda_{1}} \frac{1}{\sqrt{1-\lambda_{2}^{2}}}-\frac{\lambda_{1}}{\lambda_{2}-\lambda_{1}} \frac{1}{\sqrt{1-\lambda_{1}^{2}}}\right)$,

where

$$
\begin{aligned}
& \lambda_{1}=-\frac{\alpha z}{2\left[1+(1-\alpha) z^{T}\right]}\left[1+\left(1+\frac{8(1-\alpha) z^{T}\left[1+(1-\alpha) z^{T}\right]}{\alpha^{2} z^{2}}\right)^{1 / 2}\right] \\
& \lambda_{2}=-\frac{\alpha z}{2\left[1+(1-\alpha) z^{T}\right]}\left[1-\left(1+\frac{8(1-\alpha) z^{T}\left(1+(1-\alpha) z^{T}\right)}{\alpha^{2} z^{2}}\right)^{1 / 2}\right] .
\end{aligned}
$$


Consequently, in this particular case the generating function of the expected number of distinct sites visited is given by the closed-form expression

$$
S(z)=\left[\frac { ( 1 + ( 1 - \alpha ) z ^ { T } ) } { ( 1 - z ) ( 1 - \alpha z - ( 1 - \alpha ) z ^ { T } ) } \left[\left[\frac{\left(\lambda_{2}-\lambda_{1}\right) \sqrt{1-\lambda_{1}^{2}} \sqrt{1-\lambda_{2}^{2}}}{\lambda_{2} \sqrt{1-\lambda_{1}^{2}}-\lambda_{1} \sqrt{1-\lambda_{2}^{2}}}\right] .\right.\right.
$$

To find the large- $n$ behavior of the distinct number of sites visited we need to consider the limit $z \rightarrow 1^{-}$of the generating function. Recall that we expect a leading behavior of the form $S_{n} \sim f(\alpha) n^{1 / 2}$ because ours is basically a one-dimensional random walk, albeit intermittent. Not only do we still need to determine $f(\alpha)$, but we also need to ascertain the importance of subsequent terms. A straightforward but tedious calculation leads to the expansion

$$
S(z)=\left(\frac{2(4-3 \alpha)}{\alpha+(1-\alpha) T}\right)^{1 / 2} \frac{1}{(1-z)^{3 / 2}}-\frac{4(1-\alpha)}{\alpha^{1 / 2}(4-3 \alpha)^{1 / 2}} \frac{1}{(1-z)}+\mathcal{O}\left(\frac{1}{(1-z)^{1 / 2}}\right)
$$

Clearly, for any given value of $z$ no matter how close to unity, this "blind" expansion in powers of $(1-z)$ only makes sense if $\alpha>0$ since the second term diverges at $\alpha=0$. This is thus only a valid expansion from which asymptotic behavior can be extracted if $0<\alpha \leq 1$. At $\alpha \equiv 0$ one must go back to the original function $S(z)$, set $\alpha=0$, and then expand:

$$
S(z)=\left(\frac{2}{T}\right)^{1 / 2} \frac{1}{(1-z)^{3 / 2}}+\mathcal{O}\left(\frac{1}{(1-z)^{1 / 2}}\right) .
$$

That $\alpha=0$ is a special value that can lead to discontinuities was already noted in [20]. It is the only value of the intermittency parameter that absolutely excludes every other site from the random walk.

Inverting $S(z)$ in equations (5.4) and (5.5) at times $n=N$ we find that for the intermittent random walk with nearest-neighbour steps and jumps to next-nearestneighbour sites the leading large- $N$ behavior of $S_{N}$ is

$$
S_{N}=f(\alpha) N^{1 / 2}-c(\alpha)+\mathcal{O}\left(\frac{1}{N^{1 / 2}}\right) .
$$

Reflecting the discontinuity in $\alpha$ described above, the functions $f(\alpha)$ and $c(\alpha)$ are discontinuous at $\alpha=0$,

$$
f(\alpha)= \begin{cases}\left(\frac{8(4-3 \alpha)}{\pi(\alpha+(1-\alpha) T)}\right)^{1 / 2} & \text { for } 0<\alpha \leq 1, \\ (8 / \pi T)^{1 / 2} & \text { for } \alpha \equiv 0\end{cases}
$$

and

$$
c(\alpha)= \begin{cases}\frac{4(1-\alpha)}{\alpha^{1 / 2}(4-3 \alpha)^{1 / 2}} & \text { for } 0<\alpha \leq 1, \\ 0 & \text { for } \alpha \equiv 0 .\end{cases}
$$

Note that this "discontinuity" should be viewed with appropriate caution, since $S_{N}$ at any fixed $N$ should be a smooth function of $\alpha$. The discontinuous forms of equations (5.7) 
and (5.8) arise because we are describing an asymptotic behavior that is strictly valid only in the limit $N \rightarrow \infty$. In fact, the use of the $\alpha>0$ results is only appropriate as long as the second term in $S_{N}$ does not dominate the first. In any case, these considerations require that we recognize the $\alpha=0$ discontinuity explicitly.

The question of interest is whether $S_{N}$ has a maximum and consequently $P_{N}$ a minimum as a function of $\alpha$, that is, whether it is possible to design an optimal search strategy through the choice of the intermittency parameter. In figure 2(a) we plot the asymptotic result for the function $S_{N}$, equation (5.6), and the results of Monte Carlo simulations, for $N=10^{3}$ and $T=1$ and 2 as a function of $\alpha$. We see that indeed $S_{N}$ is a non-monotonic function of $\alpha$, and that the asymptotic form in equation (5.6) is in excellent agreement with the numerical data.

(a)

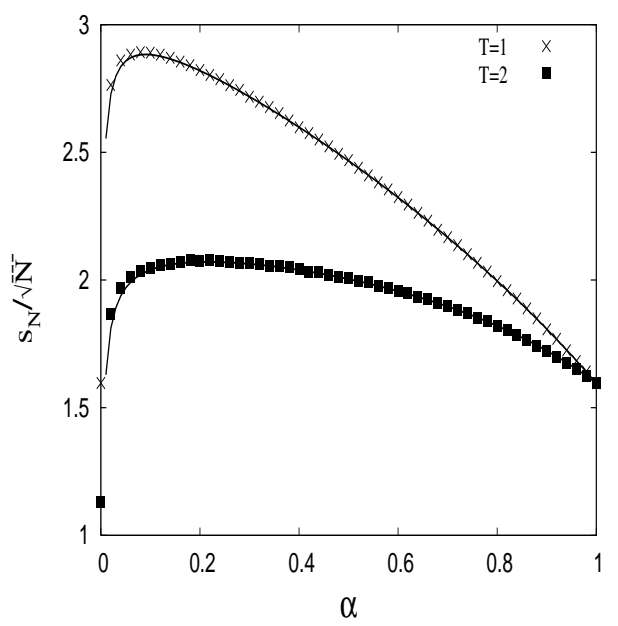

(b)

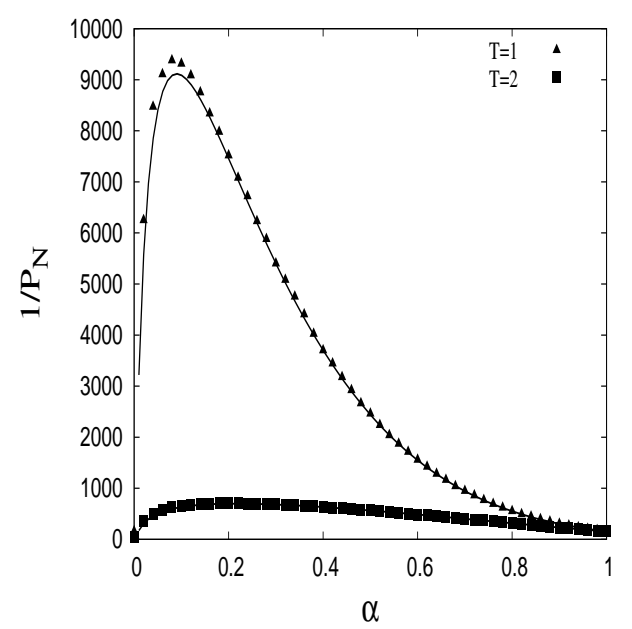

Figure 2. (a) Plot of $S_{N} / \sqrt{N}$ versus $\alpha$ at $N=1000$ for $T=1$ and $T=2$. Solid lines are analytic results, equation (5.6), while symbols denote the results of Monte Carlo simulations. (b) Plot of the inverse of the non-detection probability, $1 / P_{N}$, versus $\alpha$ for $\rho=0.1$ and $N=1000$.

Our aim is to analytically determine the value of $\alpha$ which maximizes $S_{N}$. Since we expect $\alpha>0$, we use the appropriate form of the coefficients $f(\alpha)$ and $c(\alpha)$ in equation (5.6). While the coefficient $f(\alpha)$ of the leading term in $N$ is non-monotonic, it jumps from its smallest value at $\alpha \equiv 0$ to its largest value at $\alpha=0^{+}$. For such small $\alpha$ me must retain the second term. Differentiating $S_{N}$ (both contributions) with respect to $\alpha$ to find the maximum readily leads to the optimal value

$$
\alpha_{\text {opt }}(N) \approx T\left(\frac{2 \pi}{(4-T)^{2} N}\right)^{1 / 3}
$$

with additional contributions of lower order in $N$. Consequently, the maximal expected number of sites visited by the intermittent random walk is attained at $\alpha=\alpha_{\text {opt }}(N)$ defined by equation (5.9),

$\max _{\alpha} S_{N}=\left(\frac{32 N}{\pi T}\right)^{1 / 2}-(4-T)^{1 / 3}\left(T^{1 / 6}+\frac{1}{2 T^{4 / 3}}\right)\left(\frac{32 N}{\pi T}\right)^{1 / 6}+o\left(N^{1 / 6}\right)$. 
Let us recap the ingredients of this result and analyze its significance. Because the optimal $\alpha$ for the most efficient search is positive definite for any given $N$, we needed to use the asymptotic results for $S_{N}$ that included the $c(\alpha)$ contribution. The actual asymptotic number of distinct sites visited in this optimal search, $\max _{\alpha} S_{N}=$ $(32 N / \pi T)^{1 / 2}$, is simply the value of the first term in equation (5.6) with the $\alpha>0$ form in equation (5.7), when $\alpha$ is set to zero. However, the only reason we could use this form is precisely because $\alpha$ is not identically zero. The actual contribution of the second term to $S_{N}$ turns out to be negligible, since it contributes in equation (5.9) only to order $N^{1 / 6}$, but we need the existence of this term to obtain the correct asymptotic result.

In this particular case of a search for an immobile hidden target by an intermittent random walk with nearest-neighbour and next-nearest-neighbour steps the best strategy can be summarized as follows. If we are ready to wait a sufficiently long time $N$ but want to be sure that at that moment of time we will attain the lowest possible nondetection probability $P_{N}$, we choose $\alpha=\alpha_{\text {opt }}(N)=T\left(2 \pi /(4-T)^{2} N\right)^{1 / 3}$. Note also that this strategy is quite efficient considering that nearest-neighbour and next-nearestneighbour steps are not very different from one other. The enhancement of the expected number of distinct sites visited through this strategy is a factor of $2 / \sqrt{T}$. This effect is exponentiated in the non-detection probability, so that it is dramatically apparent in figure 2(b).

\section{Optimal search strategy for arbitrary $L$}

The analysis presented in the previous section for a particular case can be generalized to arbitrary values of $L$ and $T$ by following essentially the same reasoning. The algebraic derivations that lead to the results are longer and more tedious, but the results are similar in form to those obtained previously. The outcome, as we shall see, is that the detection probability enhancement can be made much greater than that obtained under the restrictions that $L=2$ and $T=1$ or 2 . In fact, it can be improved by orders of magnitude.

As before, to calculate the generating function for the distinct number of sites visited we need to determine the generating function for the probability of being at the origin. The integral in equation (4.21) can no longer be done in closed form for arbitrary $L$ and $T$, but it is possible to carry out an expansion in powers of $(1-z)$ and retain the terms necessary for the computation of $S_{N}$. As before, the cases $0<\alpha \leq 1$ and $\alpha \equiv 0$ need to be treated separately because the latter is still a special case. For $\alpha \equiv 0$ the expansion is fairly straightforward and leads to

$$
P_{\alpha=0}(0 \mid 0 ; z)=\frac{1}{\sqrt{2 T}} \frac{1}{\sqrt{1-z}}+\mathcal{O}(\sqrt{1-z}) .
$$

The calculation is more elaborate for $0<\alpha \leq 1$. It is essential to retain not only the leading divergent contribution in $(1-z)$ as $z \rightarrow 1^{-}$, but also the contribution independent of $(1-z)$. This is because ultimately once again we need the contributions 
of $\mathcal{O}\left(N^{1 / 2}\right)$ as well as $\mathcal{O}\left(N^{0}\right)$ to $S_{N}$ to arrive at the correct optimization. We find

$P(0 \mid 0 ; z)=\frac{1}{\sqrt{2(\alpha+(1-\alpha) T)\left(\alpha+(1-\alpha) L^{2}\right)}} \frac{1}{\sqrt{1-z}}+I(\alpha, L)+\mathcal{O}(\sqrt{1-z})$,

where

$I(\alpha, L)=\frac{1}{\pi \alpha} \int_{-1}^{1} \frac{d y}{\sqrt{1-y^{2}} \sqrt{\tau_{1}^{2}-1}}\left(\frac{1-\xi_{1}^{2 L}}{1+2 \xi_{1}^{L} y+\xi_{1}^{2 L}}-\frac{L \sqrt{\tau_{1}^{2}-1}}{L^{2}\left(\tau_{1}-1\right)+(y+1)}\right)$,

and $\tau_{1}$ and $\xi_{1}$ are the values of the functions $\tau$ and $\xi$ of equation (4.20) at $z \equiv 1$. Substitution into equation (4.13) leads to

$$
\begin{aligned}
S(z)= & \left(\frac{2\left(\alpha+(1-\alpha) L^{2}\right)}{(\alpha+(1-\alpha) T)}\right)^{1 / 2} \frac{1}{(1-z)^{3 / 2}}-\frac{2 I(\alpha, L)\left(\alpha+(1-\alpha) L^{2}\right)}{(1-z)} \\
& +\mathcal{O}\left(\frac{1}{(1-z)^{1 / 2}}\right),
\end{aligned}
$$

which implies that at $n=N \gg 1$ the leading asymptotic behavior of the expected number of distinct sites visited by an intermittent random walk is

$S_{N}=\left(\frac{\left(\alpha+(1-\alpha) L^{2}\right)}{(\alpha+(1-\alpha) T)}\right)^{1 / 2}\left(\frac{8 N}{\pi}\right)^{1 / 2}-2 I(\alpha, L)\left(\alpha+(1-\alpha) L^{2}\right)+\mathcal{O}\left(\frac{1}{N^{1 / 2}}\right)$.

Finally, the integral $I(\alpha, L)$ is not available in closed form, but its important contribution to the problem can be estimated. For sufficiently large $N$ it is small everywhere except for the neighbourhood of $\alpha=0$. To extract the leading contribution for small $\alpha$ we change the variable of integration in equation (6.3) from $y$ to the auxilliary variable $u=(1+(1-\alpha) y) / \alpha$, in terms of which

$$
\begin{aligned}
& I(\alpha, L)=\frac{1-\alpha}{\pi \sqrt{\alpha}} \int_{1}^{(2-\alpha) / \alpha} \frac{d u}{(u-1) \sqrt{(u+1)(2-\alpha(u+1))}} \\
& \times\left(\frac{1-\xi_{1}^{2 L}}{(1-\alpha)\left(1+\xi_{1}^{2 L}\right)+2(\alpha u-1) \xi_{1}^{L}}-\frac{L \sqrt{u+1}}{\sqrt{u-1}\left(\alpha+(1-\alpha) L^{2}\right.}\right) .
\end{aligned}
$$

The singularity at the lower limit of integration $u=1$ is integrable for any value of $\alpha$. Furthermore, the integrand vanishes sufficiently fast to insure that it is integrable as $u \rightarrow \infty$. Hence, we may set $\alpha \equiv 0$ everywhere in the integrand and we can extend the upper integration limit to infinity. This provides the leading small- $\alpha$ contribution,

$I(\alpha, L) \sim \frac{(1-\alpha) g(L)}{\pi \sqrt{\alpha}}, \quad g(L)=\int_{0}^{\infty} \frac{d \phi}{\sinh (\phi)}\left(\operatorname{coth}(L \phi)-\frac{1}{L} \operatorname{coth}(\phi)\right)$.

The subsequent reasoning now proceeds exactly as in the previous section. We wish to determine the value of $\alpha$ that maximizes $S_{N}$. Again, we expect that this $\alpha$ is small but strictly positive, so we focus on the result (6.5). Maximizing $S_{N}$ with respect to $\alpha$ we find that

$$
\alpha_{o p t}(N) \simeq \frac{L^{7 / 3} g^{2 / 3}(L)}{V^{1 / 3}(L V-1)^{2 / 3}} \frac{1}{(8 \pi N)^{1 / 3}},
$$


The resulting asymptotic form for the distinct number of sites visited finally is

$$
\max _{\alpha} S_{N}=(L V)^{1 / 2}\left(\frac{8 N}{\pi}\right)^{1 / 2}-\gamma(L)\left(\frac{8 N}{\pi}\right)^{1 / 6}+o\left(N^{1 / 6}\right)
$$

where

$$
\gamma(L)=\frac{(4 \pi+1)}{2 \pi^{2 / 3}}\left(V g^{4}(L) L^{5}(L V-1)^{2}\right)^{1 / 6} .
$$

This is the generalization of equation (5.10). It is valid when $\alpha_{\text {opt }}(N) \ll 1$, that is, when $N \gg g^{2}(L) L^{2}$, which insures that the first term in $\max _{\alpha} S_{N}$ is dominant. Note that the leading contribution to $S_{N}$ is again the outcome of setting $\alpha=0$ in the first term of (6.5). The second term ultimately does not contribute to leading order in $N$, but, to stress once again, we were required the use the $\alpha>0$ result (6.5) because $\alpha_{\text {opt }}(N)$ is positive definite.

In figure 3(a) we plot our analytic result (6.5) using the estimate (6.7) (lines) as well as the results of Monte Carlo simulations (symbols) as a function of $\alpha$. The results are shown for $L=5$ and various values of $N$ and $T$, and the agreement is again excellent. The larger symbols in the figure denote the positions of the maxima, which are well captured by our analytical curves. Figure 3(b) shows the inverse of the associated non-detection probability for various values of $N$.

(a)

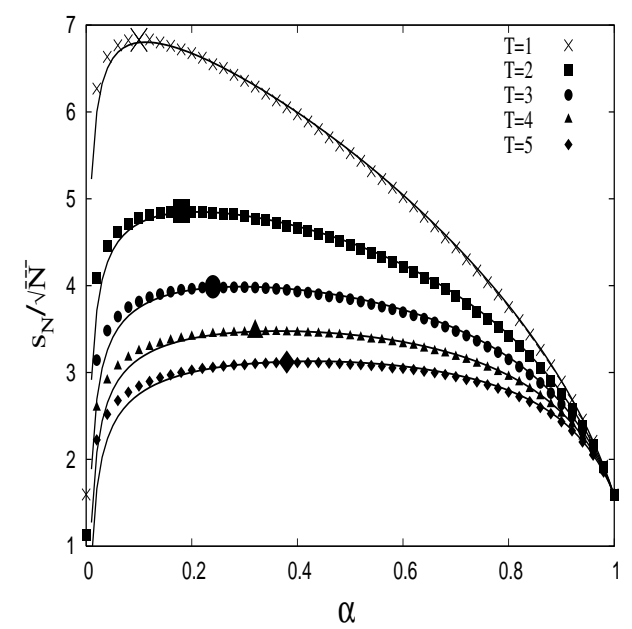

(b)

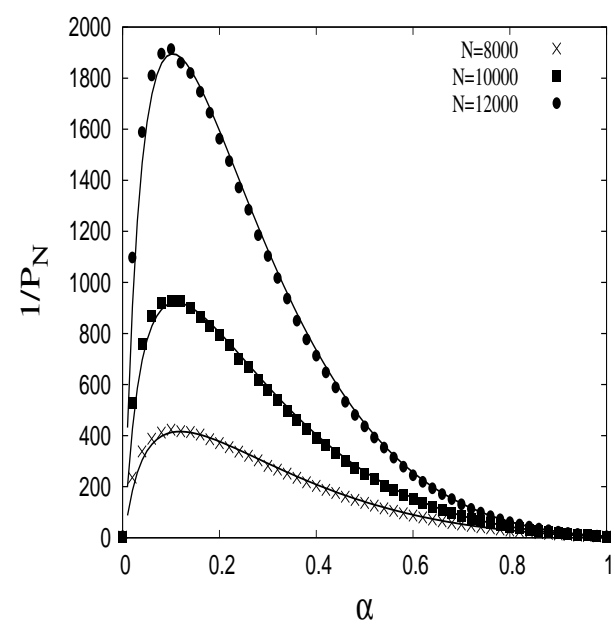

Figure 3. (a) Plot of $S_{N} / \sqrt{N}$ versus $\alpha$ for $L=5$ at $N=10000$, for $T=1,2,3,4,5$. Solid lines are analytic results, equation (6.5) with (6.7), while symbols denote the results of Monte Carlo simulations. The larger symbols denote the positions of the maxima. (b) Plot of the inverse of the non-detection probability, $1 / P_{N}$, versus $\alpha$ for $\rho=0.01$ and $N=8000,10000$, and 12000 .

The essential result of this section is the very large enhancement of the target detection probability that is possible by the appropriate $N$-dependent choice of the intermittency parameter compared to the outcome for the "pure" random walks with $\alpha=0$ or $\alpha=1$. The leading term in equation (6.9) contains the factor $\sqrt{L V}$ which 
may be large and may therefore lead to a substantial decrease in the probability that the target has not been detected up to time $N$. For example, for $L=5, T=1$, and $N=10000$, with a density of searchers as low as $\rho=0.01$ (cf. figure B(b)), the nondetection probability for the "pure" cases is $P_{N} \approx 0.2$. On the other hand, if we choose, with $\alpha=\alpha_{\text {opt }}(10000) \approx 0.07$, the probability that the target has not been yet detected up to this time is $P_{N} \approx 0.0003$, i.e., three orders of magnitude smaller. Note finally that in one dimension for fixed $L$ the optimal search strategy involves a progressively smaller fraction of nearest neighbour steps as $N$ is increased.

\section{Conclusions}

We have studied the search kinetics of a single target hidden on a one-dimensional regular lattice by a concentration of "searchers" performing intermittent random walks; that is, at each tick of the clock, each searcher has a choice of stepping on a nearest neighbour with probability $\alpha$, or flying off-lattice in either direction with velocity $V$ over a distance $L$ with probability $(1-\alpha)$. We have determined the probability $P_{N}$ that the target remains undetected up to the maximal search time $N$, have established that $P_{N}$ is a non-monotonic function of $\alpha$, and that in fact it has a sharp minimum at an $N$-dependent value of $\alpha$. Consequently, we have shown that the search efficiency can be dramatically enhanced, by orders of magnitude, by choosing $\alpha$ appropriately.

We close this paper with some observations and problems for future research. If we are at liberty to choose $L$, we may increase the number of distinct sites visited within a given time interval $N$ by picking an $N$-dependent value of $L$, cf. equation (6.9). On the other hand, equation (6.9) is a parabolic function of $L$, which implies that there should be an optimal $L$ for any fixed $N$. To wit, making $L$ too large for fixed $N$ would be counter-productive since the searchers would spend most of their time flying offlattice. On the other hand, a value of $L$ that is too small would detain the searchers in already well-explored regions and delay the exploration of new ones. This issue requires a more subtle analysis and a more intricate optimization procedure, to be discussed elsewhere [15].

Our model can be straightforwardly extended to higher dimensions. In two dimensions there is also a value of $\alpha$ that maximizes $S_{N}$ and that depends on the maximal search time $N$, as seen in the numerical results shown in figure 4 . In three dimensions and higher, we expect an $N$-independent value of $\alpha$ to maximize $S_{N}$.

The results in this paper were obtained for the case of perfect detection $(q=1)$, that is, once a searcher lands at the site occupied by the target it detects it with certainty. It is easy to show that in the general case when detection of the target upon encouter is less than certain $(0 \leq q<1)$, the probability that the target remains undetected up to time $N$ is

$$
P_{N}=\exp \left(-\rho Q_{N}\right),
$$




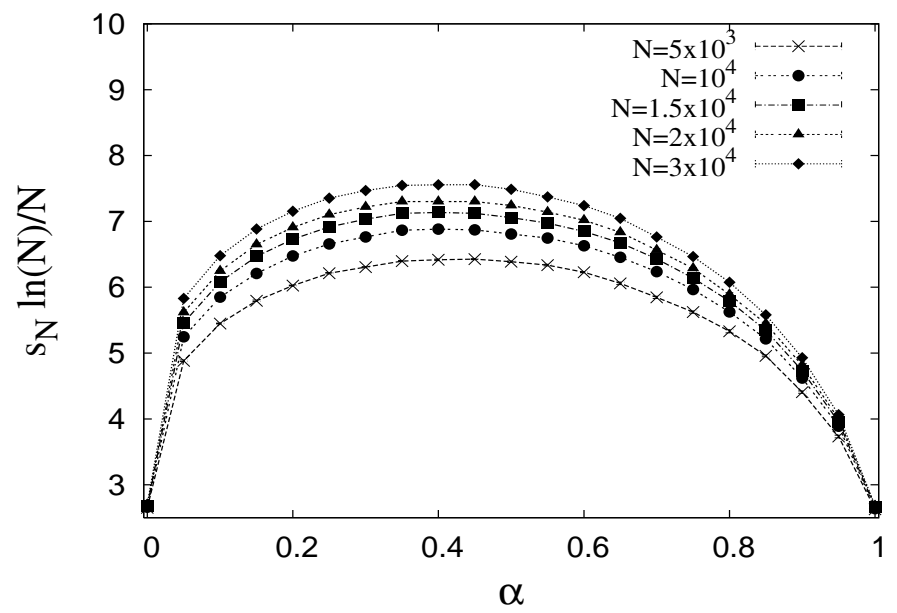

Figure 4. Results of Monte Carlo simulations on a two-dimensional square lattice. Plot of $S_{N} \ln (N) / N$ versus $\alpha$ for different $N$. Symbols denote the results of the simulations, and the solid lines are just guides for the eye.

where $Q_{N}$ is determined via its generating function,

$$
Q(z)=\sum_{N=0}^{\infty} Q_{N} z^{N}=\frac{1}{1-z} \frac{q}{[q P(0 \mid 0 ; z)+1-q]} \sum_{s} P(s \mid 0 ; z) .
$$

In low dimensional systems, i.e., in one and two dimensions, $P(0 \mid 0 ; z)$ diverges as $z \rightarrow 1^{-}$, and consequently, in the limit $N \rightarrow \infty, Q_{N}$ converges to $S_{N}$ so that the leading large- $N$ behavior will be independent of $q$ provided that $q>0$. In three and higher dimensions the leading behavior will depend on $q$.

An interesting extension of our model may be a situation in which the target itself moves randomly. It is known, however, that if this motion is diffusive (or sub-diffusive) in low dimensions, the long-time asymptotic form of the probability $P_{N}$ is exactly the same as when the target is immobile (see [22] and also [23,24]). Consequently, in low dimensions in the large- $N$ limit, the search for a diffusive (or sub-diffusive) target by searchers performing intermittent random walks will proceed in exactly the same way as determined in this paper for the case of an immobile target.

Finally, we remark that a sensible extension of this model would be to introduce the possibility of jumping, with a given velocity over arbitrary distances $l=1,2,3, \ldots$, with probability $p_{l}$. A robust approach would be to search for the distribution $p_{l}$ that minimizes the non-detection probability $P_{N}$.

\section{Acknowledgements}

The authors wish to thank D.Piorunsky, O.Bénichou, M.Moreau and R.Voituriez for stimulating discussions and useful comments, as well as O.Vasilyev for the help with numerical simulations. K.L. is supported in part by the National Science Foundation under grant PHY-0354937. 
[1] Stephens D W and Krebs J R 1986 Foraging Theory, (Princeton, NJ: Princeton University Press)

[2] Stone L D 1989 Theory of Optimal Search, (Arlington, VA, Operations Research of America)

[3] Bell J W 1991 Searching Behavior, The Behavioural Ecology of Finding Resources, (Chapman and Hall Animal Behavious Series)

[4] Frost J R and Stone L D 2001, Review of Search Theory: Advances and Applications to Search and Rescue Decision Support (US Coast Guard Research and Development Center)

[5] Klafter J, Shlesinger M, and Zumofen G 1996 Physics Today 4933

[6] Viswanathan G M et al 1999 Nature 401 911; 1996 Nature 381413

[7] Levandovsky M, Klafter J, and White B S 1988 Bull. Marine Sci. 43758

[8] Bartumeus F et al 2002 Phys. Rev. Lett. 88 097901; Phys. Rev. Lett. 89109902

[9] Raposo E P et al. 2003 Phys. Rev. Lett. 91240601

[10] Boyer D et al 2006 Proc Biol. Sci. 2731743

[11] Benichou O et al. 2005 Phys. Rev. Lett. 94, 98101; J. Phys.: Condensed Matter 17, S4275; Europhys. Lett. 70 42; see also this special issue

[12] Shlesinger M F 2006 Nature 443281

[13] O'Brien W J, Browman H I, and Evans B I 1990 American Scientist 78152

[14] Kramer D L and McLaughlin R L 2001 Amer. Zool. 41137

[15] Oshanin G, Wio H S, Lindenberg K, and Burlatsky S, in preparation

[16] Tachiya M 1983 Radiat. Phys. Chem. 21 167; Blumen A, Zumofen G, and Klafter J 1984 Phys. Rev. B 30 5379; Redner S and Kang K 1984 J. Phys. A 17 L451; Burlatsky S F and Ovchinnikov A A 1987 Zh. Eksp. Teor. Fiz. 921618 [1987 Sov. Phys. JETP 65 908]; Szabo A , Zwanzig R, and Agmon N 1989 Phys. Rev. Lett. 61 2496; Burlatsky S F, Moreau M, Oshanin G, and Blumen A 1995 Phys. Rev. Lett. 75 585; Bhatia D P, Prasad M A and Arora D 1995 Phys. Rev. Lett. 75586

[17] Benichou O, Moreau M, and Oshanin G 2000 Phys. Rev. E 613388

[18] Hughes B D 1995 Random Walks and Random Environments, (Oxford Science Publishers, Oxford)

[19] see, e.g., Lorenzutta $S$ et al. 1995 Rendiconti di Matematica 15405

[20] Lindenberg-Lakatos K and Shuler K E 1971 J. Math. Phys. 12633

[21] Lindenberg K 1972 J. Stat. Phys. 10485

[22] Bray A J and Blythe R A 2002 Phys. Rev. Lett. 89 150601; Blythe R A and Bray A J 2003 Phys. Rev. E 67041101

[23] Oshanin G, Benichou O, Coppey M, and Moreau M 2002 Phys. Rev. E 66 060101; Moreau M, Oshanin G, Benichou O, and Coppey M 2004 Phys. Rev. E 69046101

[24] Yuste S B and Lindenberg K 2005, Phys. Rev. E 72061103 